\title{
Charging electric vehicles, baking pizzas, and melting a fuse in Lochem
}

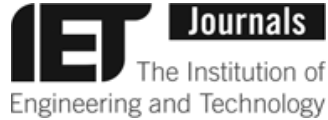

ISSN 2515-0855 doi: 10.1049/oap-cired.2017.0340 www.ietdl.org

\author{
Gerwin Hoogsteen ${ }^{1} \bowtie$, Albert Molderink ${ }^{1}$, Johann L. Hurink ${ }^{1}$, \\ Gerard J.M. Smit ${ }^{1}$, Ben Kootstra ${ }^{2}$, Friso Schuring ${ }^{2}$ \\ ${ }^{1}$ Department of Electrical Engineering, Mathematics and Computer Science, University of \\ Twente, Enschede, The Netherlands \\ ${ }^{2}$ Liandon, Duiven, The Netherlands \\ 凶-mail: g.hoogsteen@utwente.nl
}

\begin{abstract}
To evaluate the impact of the energy transition on distribution grids, a year 2025 scenario stress test was conducted in a real Dutch distribution grid. For this, the authors confronted the local low-voltage grid of a village with 20 electrical vehicles and ovens. The result was a short service interruption which could not be avoided by control. Furthermore, severe unbalance and high peak load were observed, resulting in large voltage drops and severe neutral point shifts. The obtained measurements are used to validate simulation models used to study the effect of novel control strategies to balance the local grid.
\end{abstract}

\section{Introduction}

The rapid adoption and integration of distributed energy resources (DERs) in the low voltage (LV) grid over the last few years has resulted in a vast amount of research on the so-called smart grid. This change is driven by climate change and the goal to reduce the dependence on fossil fuels from unstable regions. With smart grid technology, energy usage and production of DERs such as electric vehicles (EVs), rooftop photovoltaic (PV), and heat pumps (HPs) can be coordinated. Equipped with communication technology, the smart grid is able to coordinate the inherent flexibility that these DERs can offer to perform tasks such as peak shaving and thereby alleviate stress and avoid overloading of the grid. To this end, smart grids are seen as a cost-effective alternative to conventional upgrades, such as installing new cables and transformers.

The smart grid paradigm is a heavily studied subject in the academic world. Numerous models and optimisation algorithms are proposed to study smart grid solutions, such as surveyed by Siano [1]. Simulation studies, such as [2], point out that the electrification challenges physical grids and that localised control is required to manage both the global energy profile and ensure a proper power quality. In addition to simulation studies, field tests are conducted to test smart grid solutions in practice. One of these field test sites is the Dutch town of Lochem. Previously, an experiment was conducted in this test site to measure the effects of further electrification. The results, published in [3], showed that there is a serious risk of unbalanced loading in the grid.

This paper describes a follow-up test in which we created a year 2025 scenario via load projections. In this so-called 'stress test' experiment, we aimed to identify weak points in an existing grid, such that smart grid technology can be tailored to avoid related problems. Within the stress test, we used the PowerMatcher smart grid coordination technology [4] to perform peak shaving and investigate if current concepts provide enough controllability in demanding, unbalanced situations.

The remainder of the paper is organised as follows. The following section describes the test site and stress test set-up. Subsequently, we analyse the obtained measurements and use these measurements to validate simulation models. Based on our observations, we start a discussion and give recommendations on smart grid operation. The paper ends with conclusions.

\section{Set-up}

The test site consists of three parts. First, we describe the layout of the residential area, its LV grid, and the available loads to create a 2025 scenario. Secondly, we discuss the PowerMatcher set-up and lastly, the script for the stress test is presented.

For this stress test, we considered the designated smart grid test site in Lochem. The goal of the test site is to investigate smart grid technologies and the impact of high penetrations of rooftop PV and electric mobility usage. Therefore, metering equipment is installed at the substation and in households. Furthermore, two controllable three-phase AC charging station points are installed in the grid. One station allows one car to charge at $22 \mathrm{~kW}$, and the second is able to charge two cars simultaneously at $22 \mathrm{~kW}$ each. In addition to privately owned full electric vehicles (FEVs) and plug-in hybrid electric vehicles (PHEVs), the local energy corporation (LochemEnergie) also rents FEVs to participants. Furthermore, many households have installed rooftop PV. The LV grid consists of one feeder with a maximum distance of $700 \mathrm{~m}$ between the MV/LV substation and a serviced household. This network services 80 households with a mix of apartments, detached homes, and terraced homes. The feeder is protected by three $160 \mathrm{~A}$ Weber $500 \mathrm{~V}$ gFF fuses at the transformer to meet safety regulations.

We aimed to create a year 2025 scenario based on current projections and expectations on electric loads. We used projections from [5] for electric mobility in the Netherlands, which indicate a $12.5 \%$ penetration of electric mobility in 2025 . Combined with local mobility numbers from [6], we find that, with 132 cars in this neighbourhood, $16.5 \mathrm{EVs}$ are to be expected in this grid (assuming that the number of cars does not change). For the stress test, we had a total of 20 EVs (a mix of FEVs and PHEVs) at our disposal. Furthermore, an increasing electrification of cooking and heating is expected in the Netherlands. For this, pizzas to be baked in electric ovens and additional loads were available to be added to the grid during the stress test. Finally, additional power quality measurement equipment was installed at both the transformer and at the controllable charging stations installed at the end of the feeder.

These charging stations have a three-phase connection with a maximum power of $22 \mathrm{~kW}$ to each car. The DSO (Alliander) is able to control the charging stations individually through an API. In our case, we tested the control of the charging. As neither the PowerMatcher [4] nor the charging stations can control the three 
phases independently, we fed data of the highest loaded phase as measured at the transformer to the PowerMatcher. The control system is programmed to perform peak shaving at a predefined maximum power value. Based on the measured value, the system decides to lower or increase the allowed charging. Owing to other technical limitations of both the charging equipment and various EVs, only one control action with a maximum of $500 \mathrm{~W}$ per phase could be performed per minute. Experiments have shown that these settings result in a stable control system. With other settings, the control system experienced instability and control actions would be neglected. As the fuses can sustain overcurrent for quite some time, we have set the maximum power to $45 \mathrm{~kW}$ ( $\sim 196 \mathrm{~A})$ per phase. The fuse can sustain a current of $250 \mathrm{~A}(57.5 \mathrm{~kW})$ for $\sim 2 \mathrm{~h}$ according to the datasheet.

The stress test was made possible with the help of the strongly involved local population and energy corporation LochemEnergie. Party tents, displays, and measurement equipment were set up, such that everybody was able to witness the developments of the field test. The script for the stress test was follows:

- Before 7:00 PM: We started with a baseline measurement. Based on historical data, the total load was expected to $\sim 55 \mathrm{~kW}$.

- 7:00 PM: We connected three FEVs at the $22 \mathrm{~kW}$ charging stations one by one.

- 7:30 PM: We increased the load by connecting 17 additional EVs at domestic wall sockets. At this point, we approximate the expected load in 2025.

- 7:45 PM: The 20 pizzas were distributed over the households to be baked in electric ovens.

- 8:00 PM: All participants were encouraged to consume as much power as possible for the test.

\section{Stress test results}

This section discusses the results as plotted in Figs. 1-4 in chronological order. We further note that the voltage at the MV/ $\mathrm{LV}$ transformer was stable during the stress test at an average of $238.7 \mathrm{~V}$ with a standard deviation of $0.6 \mathrm{~V}$.

\subsection{7:00 PM - start of the stress test}

The interest in the experiment by the local population (they gathered at the tent) was a reason why we did not reach the expected $55 \mathrm{~kW}$ by 7:00 PM as shown in Figs. 1 and 2. Just before 7:00 PM, the total load was $\sim 45 \mathrm{~kW}$. At this point, the power factor averages at 0.96 , resulting in three overlapping lines in Fig. 2. The load in the network was adequately balanced, which also resulted in a normal neutral point shift between 2 and $3 \mathrm{~V}$ at the charging station (Fig. 3).

At 7:00 PM, we connected the first FEV (a Tesla Model S) to charging station (up to $22 \mathrm{~kW}$ ). This is clearly visible with an increase in the load by $21 \mathrm{~kW}$. This increase resulted in a voltage drop of $5.0 \mathrm{~V}$ on average (from 234.7 to $229.7 \mathrm{~V}$ ) at the charging station. The Tesla adds a bit of reactive power (2.7 kvar, Fig. 4), but improves the overall power factor to an average of 0.99 .

At 7:15 PM, we connected a second FEV (a Renault ZOE) to one of the charging stations, resulting in another jump of $\sim 20 \mathrm{~kW}$. The Tesla simultaneously shows a short power dip for $11 \mathrm{~s}$ on phases 1 and 3 which is reported as transient by our measurement equipment. The load increase is slightly lower and the resulting voltage drop is $4.5 \mathrm{~V}$ (from 230.6 to $227.1 \mathrm{~V}$, Fig. 3).

A couple minutes later, we tried to connect the third FEV to the second charging station. However, the system was failing and refused to charge this car. Thus, we ended up with only two controllable FEVs. At 7:23 PM, the Tesla Model S suddenly stopped charging for no apparent reason. At 7:35 PM, we connected another car (a second Renault ZOE) at this charging station, resulting in the jump of $20 \mathrm{~kW}$ as seen in Fig. 1.

\subsection{7:28 PM - connecting 17 additional EVs}

In the meanwhile, at 7:28 PM and onwards, the remaining $17 \mathrm{EVs}$ were connected to wall sockets or privately owned charging stations in the street. We measured a rapid, but not instantaneous, increase from 65 to $116 \mathrm{~kW}$. This increase of $51 \mathrm{~kW}$ equals $3 \mathrm{~kW}$ per EV on average. The PowerMatcher started to reduce the maximum charging power in steps of $500 \mathrm{~W}$ per phase during this load increase. This is also visible in Fig. 2 where the green line, indicating the load when no control would have been applied, starts to differ from the measured power. The total load does not drop as quickly by the control actions as would be desired due to the limitations mentioned.

Also from this point on, the expected unbalance between the three phases becomes visible in Fig. 1. It results in a slight neutral point shift to $5.0 \mathrm{~V}$ as shown in Fig. 3 due to the significantly higher load on phase 3 in comparison to phases 1 and 2 . This also resulted in a higher voltage drop on phase 3 at the charging station, while phase 2 experiences a higher voltage at $\sim 7: 40$ PM $(230.2 \mathrm{~V})$ compared with $\sim 7: 20 \mathrm{PM}(227.1 \mathrm{~V})$, despite a slightly higher load (33.9 versus $31.3 \mathrm{~kW})$ and the same voltage at the transformer for phase $2(239.2 \mathrm{~V})$.

\subsection{7:45 PM - baking pizzas}

At 7:45 PM, the ovens were switched on to bake the pizzas, which resulted in load increase from $\sim 130$ to $153 \mathrm{~kW}$ at the transformer. The PowerMatcher reduced the maximum charging power by $1.6 \mathrm{~kW}$ per car during the activations of ovens. The total effect of the ovens is therefore $\sim 16 \mathrm{~kW}$ of additional load (Fig. 2). The dip in power consumption at around 7:48 PM is due to the two EVs connected to the controllable charging stations that stopped charging. The ovens restored the balance between the phases slightly, while the load on phase 3 experiences a sudden drop.

The three-phase charger of the Renault ZOE showed interesting behaviour. The amount of reactive power steadily increased as the active power consumption decreased. At 7:48 PM, the maximum charging power dropped under $5 \mathrm{~kW}$ and as a result, the car stopped charging. Remarkable is that the Renault stopped charging after the reactive power was larger than the active power (power factor $<0.707$ ). Even after it stopped charging, it still drew reactive power $(6.3 \mathrm{kvar})$ from the grid.

\subsection{Maximum load}

Earlier than anticipated, the volunteers maxed out their household power consumption by switching on other loads, ending up at a rather stable load of $150-160 \mathrm{~kW}$ around 8:00 PM. From this point on, the unbalance between the phases increased again to a point where phase 3 was loaded with $25.7 \mathrm{~kW}$ more than the average load on the other two phases at 7:52 PM (Fig. 1). At this point, the load on phase 3 was $69.0 \mathrm{~kW}$ compared with 43.3 and $43.1 \mathrm{~kW}$ for phases 1 and 2, respectively. The maximum neutral point shift measured was $7.4 \mathrm{~V}$ and the voltage for phase 3 was $207.0 \mathrm{~V}$, which is a $31.0 \mathrm{~V}$ voltage drop between the transformer and the charging station. The observed peak load was $171 \mathrm{~kW}$, significantly above the capacity limit of the fuse of $110 \mathrm{~kW}$ ( $37 \mathrm{~kW}$ per phase).

The last load increase is due to the Tesla Model S (which stopped charging earlier on) being reconnected to release its anti-theft protected charging cord. This resulted in a maximum total load of $194 \mathrm{~kW}(76.8 \mathrm{~kW}$ on phase 3) and voltage of $206.0 \mathrm{~V}$ on phase 3 at the charging station. This last increase was the final push that the fuse protecting phase 3 required to melt after being heavily overloaded for a substantial amount of time. The loss of one phase also resulted in an increase in the neutral to ground voltage to 48.9 V. Fig. 2 also shows that the apparent power suddenly increases as a result. The resulting power factor is 0.81 and the reactive power is $64.8 \mathrm{kvar}$. The interruption was resolved within $5 \mathrm{~min}$. 


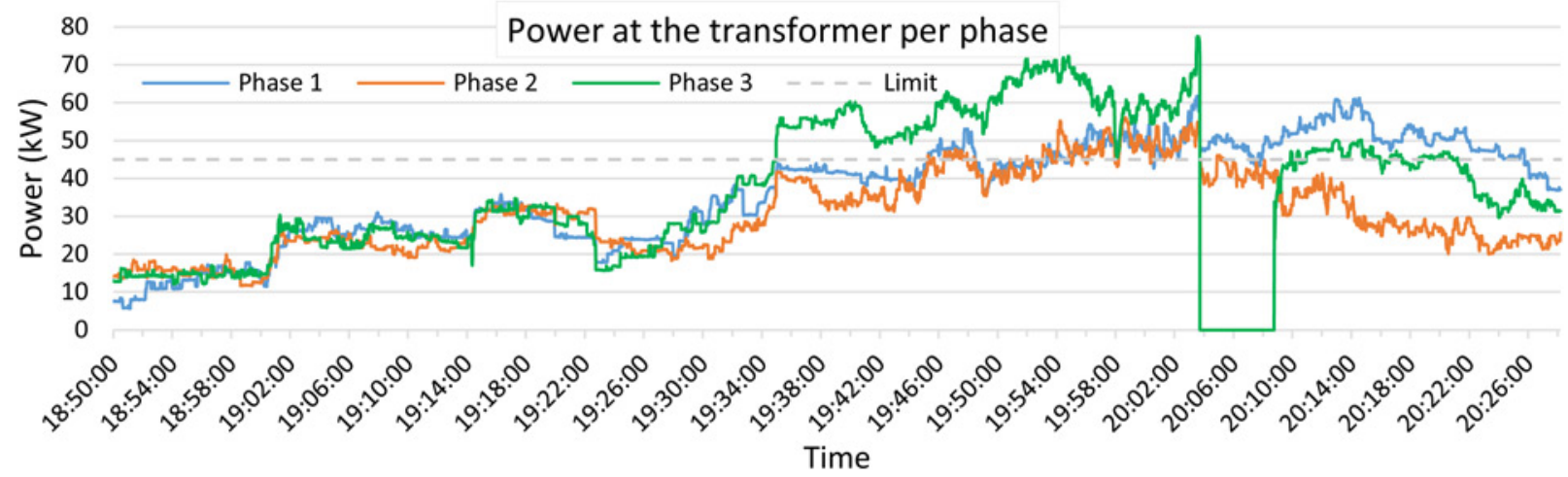

Fig. 1 Power measured at the transformer per phase (solid) and smart charge limit (dashed)

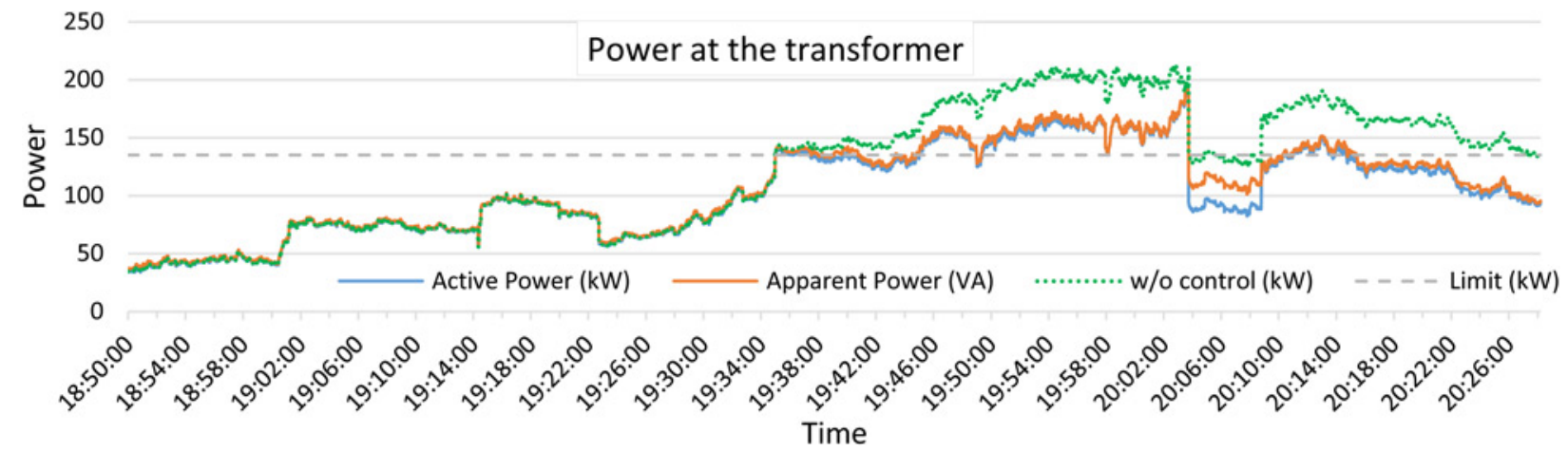

Fig. 2 Total active and reactive power at the transformer. The green line indicates the expected value without smart charging

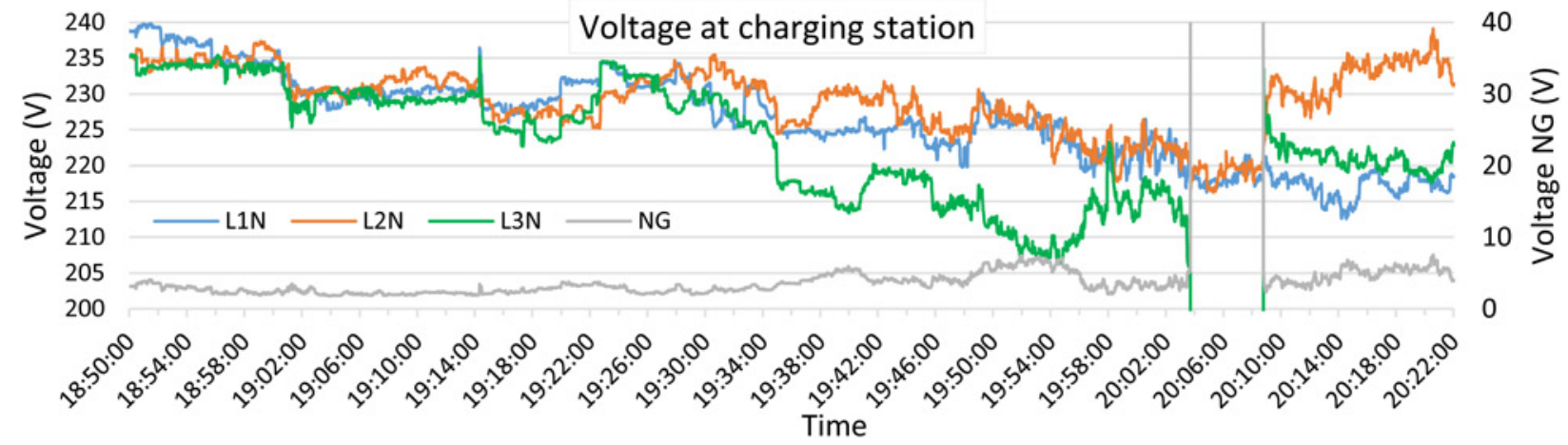

Fig. 3 Voltage measured at the charging station between the three phases and neutral and between neutral and ground (grey, right axis)

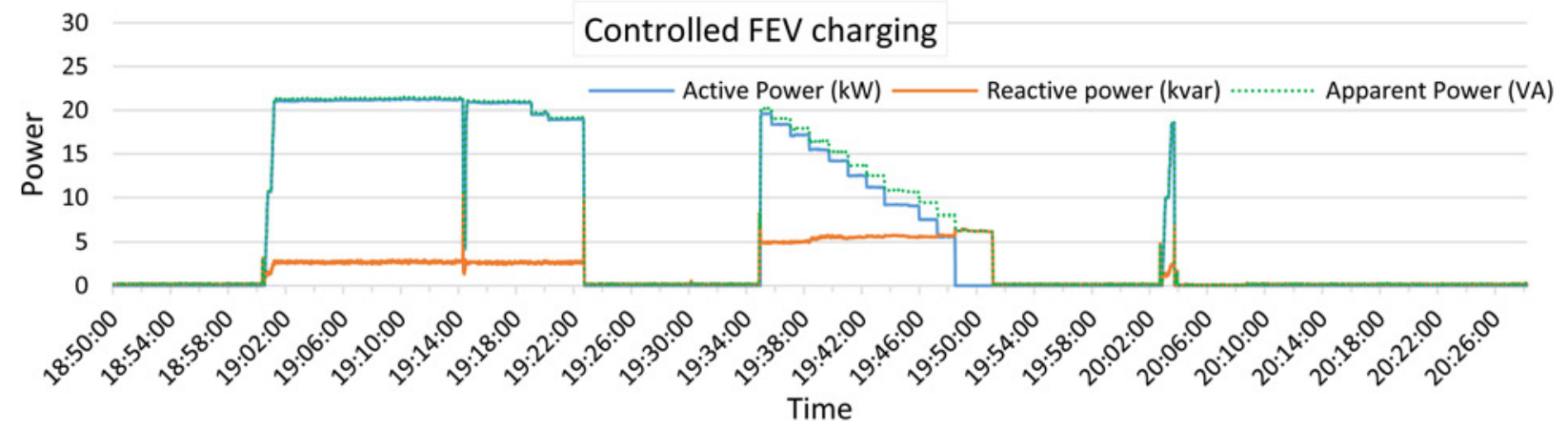

Fig. 4 Power measured at one of the charging stations 


\section{Simulation results}

As it asks quite some effort to carry out field tests, it is important to have simulation tools, which can be used to simulate scenarios without executing them in practice. A time network model with high accuracy for the neighbourhood of the stress test and an unbalanced three-phase AC load-flow simulation module for the smart grid simulation tool developed at the University of Twente are used. In previous work [2] and [3], we already conducted simulations and validations under normal circumstances. With the measurement data obtained in this stress test, we can also validate the simulation accuracy under heavy loads and in unbalanced situations.

Voltage measurement data obtained at the transformer is imported and used to set the reference voltage at the slack bus of the grid model. The power drawn by the controllable charging stations is added to the model at their respective positions in the grid using a constant power model. For the household loads, we need to make some assumptions since not all households participate in the metering project and available data is anonymous for privacy reasons. Hence, we used estimations of the household load profiles based upon measurement data. We first observe that the substantial load from the fast chargers is known. Secondly, we have knowledge of the charging location of each of the 17 EVs that were connected to domestic wall sockets. Each of these EVs was charging at a rate of $3 \mathrm{~kW}$ on average, which makes up $51 \mathrm{~kW}$. Furthermore, most of the 20 pizzas were baked in these homes. We add these loads to the model at their respective point using a constant power model. Thirdly, we expect the base load to remain rather constant and evenly distributed among the households, which makes up $45 \mathrm{~kW}$ of load. For each house, we spread the load across the three phases based on the respective fraction of the load measured at the transformer.

The goal is to compare the simulation results with the measurement data. We note that we can add these identified loads to their location in the model with a relatively high accuracy. A correct placement of the loads is important to ensure that the correct currents run through the cables in the model, which result in the voltage drops as measured during the stress test. Therefore, the time interval between reconnecting the second Renault ZOE (7:35 PM) and just before the population started to bake pizzas (7:45 PM) has the highest accuracy. Also the time frame between 7:45 PM and the outage has a relative high accuracy.

Fig. 5 shows the results of both the measurements (darker and thinner lines) and simulation results (light thicker lines). It is clear that the simulated voltages match the measurements accurately, especially in the indicated time frame between 7:35 and 7:45 PM. For the time frame between 7:35 and 7:45 PM, we find an average deviation of $0.55 \mathrm{~V}$ with a standard deviation of $0.82 \mathrm{~V}$. The maximum deviation was $+2.64 \mathrm{~V}$ and the minimum $-1.95 \mathrm{~V}$. For the depicted time frame from 7:30 to 8:00 PM, these values are an average of $1.51 \mathrm{~V}$ and a standard deviation of $1.54 \mathrm{~V}$. These

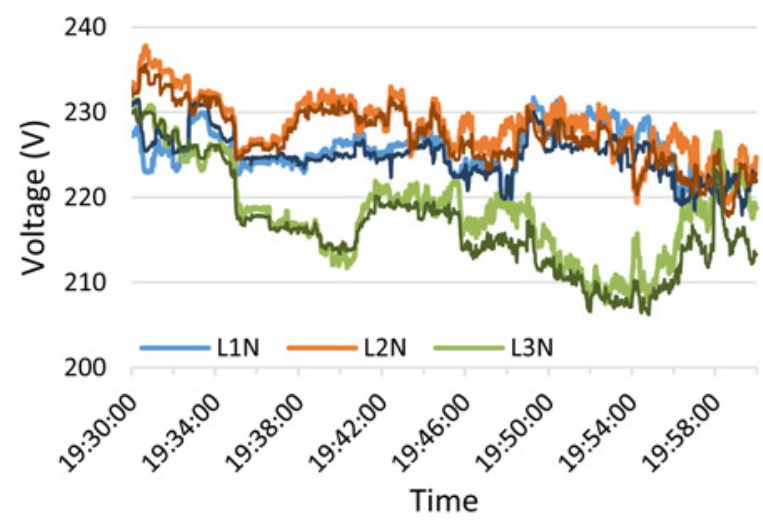

Fig. 5 Voltages obtained for the three phases in post-simulation (ight lines as in the legend) and measurements (darker lines) simulation results give the confidence that simulation studies for this grid, or comparable grids in the Netherlands, can be used to get accurate results on what to expect in futuristic scenarios. In addition, these grid models can be used to investigate how smart grid control systems behave under various circumstances in a cyber-physical simulation environment.

\section{Discussion}

The stress test has led to a short outage after heavily overloading a fuse for $\sim 30 \mathrm{~min}$ based on a 2025 scenario. With this result, it is safe to conclude that control is required to exploit flexibility to avoid outages. We denote that loads from ovens and the population maxing out their load were not directly part of the 2025 scenario. However, it is not unlikely to see such high loads at certain moments in time. Especially with the electrification, where heating is done by HPs and cooking through induction stoves, while the load synchronises with people arriving home from work.

One important observation during the stress test is the severe unbalance that occurred. This unbalance caused the fuse of phase 3 to be heavily overloaded (compared with the other two phases) for a substantial amount of time. The other two phases were kept close to the power limit that was set, which was enough to keep those fuses from melting. During the test, we had no means to control the load on individual phases. Furthermore, it is not documented to which phase(s), a household and large loads are connected. Hence, we advise to research possibilities to detect and control the load on individual phases. By restoring the balance, additional capacity may be usable in grids while voltage levels improve. Large loads like EVs could be important elements in balancing the load over the phases. For this, the charging stations then also have the possibility to spread the load over the three phases as desired by a steering signal. The diversity of controllability and effects on the grid is another issue. We encountered troubles with reducing the load of a Tesla Model S while the Renault ZOE showed a power consumption-dependent power factor. More standardisation and communication about supported functionality by equipment is required to optimise the control actions with respect to control and grid stability.

Lack of controllability behind the metre (in the consumers' premises) is also identified as a problem. While we had control over the charging poles, the uncontrollable load of the consumers caused most trouble. Therefore, without incentives to perform load shifting at house level, the control of assets owned by the DSO have little value as they do not resolve expected problems completely.

\section{Conclusions}

A stress test was conducted to create a year 2025 scenario in an existing residential area. The peak load coming from EVs, ovens, and additional loads led to a short supply interruption. The overall conclusion is that control and consumer incentives are necessary to avoid such outages in the future. Also the lack of control over the three phases resulted in severe unbalance. Potential improvements lie in a better spread of loads over the three phases by bookkeeping and control. With a better spread of load across phases, the available grid capacity can be optimised and thereby costs of conventional grid reinforcements can be avoided while maintaining a high quality of service.

The simulation results based on the scenario of the stress test show that the model is accurate. For future work, this simulation environment can be used to study effects of advanced control mechanisms that tackle the problems of unbalanced loads.

\section{Acknowledgments}

This research is conducted within the IPIN programme supported by the Netherlands Enterprise Agency and within the EASI project 
(\#12700) supported by STW and Alliander. The authors would like to thank LochemEnergie and all volunteers to make this stress test possible.

\section{References}

1 Siano, P.: 'Demand response and smart grids - a survey', Renew. Sustain. Energy Rev., 2014, 30, pp. 461-478
2 Hoogsteen, G., Molderink, A., Hurink, J.L., et al.: 'Managing energy in time and space in smart grids using TRIANA'. Proc. IEEE PES Innovative Smart Grid Technologies, Europe, Istanbul, 2014

3 Hoogsteen, G., Molderink, A., Hurink, J.L., et al.: 'Impact of peak electricity demand in distribution grids: a stress test'. PowerTech, 2015 IEEE Eindhoven

4 Kok, K.: 'The PowerMatcher: smart coordination for the smart electricity grid', PhD dissertation, Vrije Unviversiteit Amsterdam, 2013

5 Netherlands Enterprise Agency: 'Electric mobility gets up to speed, 2011-2015 action plan', 2011

6 Centraal Bureau voor de Statistiek: 'Bedrijfsvoertuigen, personenauto's, motoren; aantal/1000 inwoners, regio's', 2016 